\title{
Some new estimates for Fejér type inequalities in quantum analysis
}

\author{
Kamel Brahim, Latifa Riahi and Muhammad Uzair Awan
}

\begin{abstract}
In this paper we derive some new quantum estimates of Fejér type inequalities which involve Riemann type of quantum integrals via some classes of convex functions. We also discuss some special cases which can be deduced from the main results of this paper.
\end{abstract}

Mathematics Subject Classification (2010): 26D15, 26A51.

Keywords: Convex function, $s$-convex function, $h$-convex function, $m$-convex function, $(s, m)$-convex function, Riemann-type $q$-integral, $q$-Jackson integral.

\section{Introduction}

A function $f: I \subseteq \mathbb{R} \rightarrow \mathbb{R}, I$ is an interval, is said to be a convex function on $I$ if

$$
f(t x+(1-t) y) \leq t f(x)+(1-t) f(y)
$$

holds for all $x, y \in I$ and $t \in[0,1]$. If the reversed inequality in (1.1) holds, then $f$ is said to be concave. Let $f: I \subseteq \mathbb{R} \rightarrow \mathbb{R}$ be a convex function defined on the interval $I$ and $a, b \in I$ with $a<b$. Then the following double inequality holds:

$$
f\left(\frac{a+b}{2}\right) \int_{a}^{b} p(x) d x \leq \int_{a}^{b} f(x) p(x) d x \leq \frac{f(a)+f(b)}{2} \int_{a}^{b} p(x) d x,
$$

where $p:[a, b] \rightarrow \mathbb{R}$ is non-negative, integrable, and symmetric about $x=\frac{a+b}{2}$. This inequality is known as the Fejér inequality for convex functions (see $[2,3,16,17]$ ).

Theory of convexity plays an important role in different fields of pure and applied sciences. Due to its importance in recent years several new generalizations of classical convexity have been proposed in the literature. Breckner [1] introduced the notion of $s$-convex function, as

Definition $1.1([1])$. Let $s \in(0,1]$. A function $f:[0, \infty) \rightarrow[0, \infty)$ is said to be $s$-convex in the second sense if

$$
f(t x+(1-t) y) \leq t^{s} f(x)+(1-t)^{s} f(y)
$$


for all $x, y \in[0, \infty)$ and $t \in[0,1]$. This class of $s$-convex functions is usually denoted by $K_{s}^{2}$.

For more information on $s$-convex functions, see [4].

In 2007, Varošanec [15] introduced the notion of $h$-convex functions, which not only generalizes the class of convex functions but also some other classes of convex functions, see [15]. Thus it was noticed that the class of $h$-convex functions is quite unifying one. This class is defined as:

Definition $1.2([15])$. Let $h: J \subseteq \mathbb{R} \rightarrow \mathbb{R}$ be a positive function and $[0,1] \subset J$. We say that $f: I \subseteq \mathbb{R} \rightarrow \mathbb{R}$ is $h$-convex function, or that $f$ belong to the class $S X(h, I)$, if $f$ is nonnegative and for all $x, y \in I$ and $t \in(0,1)$ we have

$$
f(t x+(1-t) y) \leq h(t) f(x)+h(1-t) f(y) .
$$

If inequality (1.4) is reversed, then $f$ is said to be $h$-concave, i.e. $f \in S V(h, I)$. Obviously, if $h(t)=t$, then all nonnegative convex functions belong to $S X(h, I)$ and all nonnegative concave functions belong to $S V(h, I)$; and if $h(t)=t^{s}$, where $s \in(0,1)$, then $S X(h, I) \supseteq K_{s}^{2}$.

In [13], G. H. Toader defined the concept of $m$-convexity as the following:

Definition 1.3. The function $f:[0, b] \rightarrow \mathbb{R}$ is said to be $m$-convex, where $m \in[0,1]$, if for every $x, y \in[0, b]$ and $t \in[0,1]$ we have:

$$
f(t x+m(1-t) y) \leq t f(x)+m(1-t) f(y) .
$$

Denote by $K_{m}(b)$ the set of the $m$-convex functions on $[0, b]$.

In [6], V. G. Miheşan introduced the class of $(s, m)$-convex functions as the following:

Definition 1.4. The function $f:[0, b] \rightarrow \mathbb{R}$ is said to be $(s, m)$-convex, where $(s, m) \in$ $(0,1]^{2}$, if for every $x, y \in[0, b]$ and $t \in[0,1]$ we have

$$
f(t x+m(1-t) y) \leq t^{s} f(x)+m\left(1-t^{s}\right) f(y) .
$$

Denote by $K_{m}^{s}(b)$ the set of the $(s, m)$-convex functions on $[0, b]$.

In [17], Yang and Tseng established the following theorem

Theorem 1.5 (see [17], Remark 6). Let $f:[a, b] \rightarrow \mathbb{R}$ be a convex function and $p:[a, b] \rightarrow \mathbb{R}$ be a nonnegative, integrable and symmetric about $x=\frac{a+b}{2}$. If $H$ and $F$ are defined on $[0,1]$ by

$$
H(t)=\int_{a}^{b} f\left(t x+(1-t) \frac{a+b}{2}\right) p(x) d x
$$

and

$F(t)=\int_{a}^{b} \frac{1}{2}\left[f\left(\frac{1+t}{2} a+\frac{1-t}{2} x\right) p\left(\frac{x+a}{2}\right)+f\left(\frac{1+t}{2} b+\frac{1-t}{2} x\right) p\left(\frac{x+b}{2}\right)\right] d x$,

then $H, F$ are convex and increasing on $[0,1]$ and for all $t \in[0,1]$

$$
f\left(\frac{a+b}{2}\right) \int_{a}^{b} p(x) d x=H(0) \leq H(t) \leq H(1)=\int_{a}^{b} f(x) p(x) d x
$$


and

$$
\int_{a}^{b} f(x) p(x) d x=F(0) \leq F(t) \leq F(1)=\frac{f(a)+f(b)}{2} \int_{a}^{b} p(x) d x .
$$

In [12] , M. Z. Sarikaya, E. Set and M. E. Özdemir established the following inequality:

Theorem 1.6. Let $f \in S X(h, I), a, b \in I$ with $a<b, f \in L_{1}([a, b])$ and $p:[a, b] \rightarrow \mathbb{R}$ is nonnegative, integrable and symmetric about $\frac{a+b}{2}$. Then, for $h\left(\frac{1}{2}\right) \neq 0$, we have

$$
\begin{aligned}
\frac{1}{2 h\left(\frac{1}{2}\right)} f\left(\frac{a+b}{2}\right) \int_{a}^{b} p(x) d x & \leq \int_{a}^{b} f(x) p(x) d x \\
& \leq \frac{f(a)+f(b)}{2}(h(t)+h(1-t)) \int_{a}^{b} p(x) d x .
\end{aligned}
$$

In [14], the following inequalities of Fejér type via $s$-convex function was derived:

Theorem 1.7. Let $f \in K_{s}^{2}, a, b \in[0, \infty[$ with $a<b$ and $p:[a, b] \rightarrow \mathbb{R}$ is nonnegative, integrable and symmetric about $\frac{a+b}{2}$. Then

$$
\begin{aligned}
2^{s-1} f\left(\frac{a+b}{2}\right) \int_{a}^{b} p(x) d x & \leq \int_{a}^{b} f(x) p(x) d x \\
& \leq \frac{f(a)+f(b)}{2} \int_{a}^{b}\left(\left(\frac{b-x}{b-a}\right)^{s}+\left(\frac{x-a}{b-a}\right)^{s}\right) p(x) d x
\end{aligned}
$$

Again, in [14], the authors proved the following theorems:

Theorem $1.8([14])$. Let $f:[0, \infty[\rightarrow \mathbb{R}$ be an $m$-convex function with $m \in] 0,1]$. If $0 \leq a<b<\infty$ and $f \in L_{1}[0, b]$, then

$$
\int_{a}^{b} f(x) p(x) d x \leq \min \left\{\frac{f(a)+m f\left(\frac{b}{m}\right)}{2}, \frac{f(b)+m f\left(\frac{a}{m}\right)}{2}\right\} \int_{a}^{b} p(x) d x .
$$

Theorem 1.9 ([14]). Let $f:[0, \infty[\rightarrow \mathbb{R}$ be an $m$-convex function with $m \in] 0,1]$. If $0 \leq a<b<\infty$ and $f \in L_{1}[0, b]$, then

$$
\begin{aligned}
& f\left(\frac{a+b}{2}\right) \int_{a}^{b} p(x) d x \leq \int_{a}^{b} \frac{f(x)+m f\left(\frac{x}{m}\right)}{2} p(x) d x \\
\leq & \frac{1}{8}\left(f(a)+f(b)+2 m\left(f\left(\frac{a}{m}\right)+f\left(\frac{b}{m}\right)\right)+m^{2}\left(f\left(\frac{a}{m^{2}}\right)+f\left(\frac{b}{m^{2}}\right)\right)\right) \int_{a}^{b} p(x) d x .
\end{aligned}
$$

The aim of this work is to establish the $q$-analogue of Fejér inequalities for some convex type functions. For this we recall some basic concepts of quantum calculus. Let $0<q<1$, the $q$-Jackson integral from 0 to $b$ is defined by [5] as:

$$
\int_{0}^{b} f(x) d_{q} x=(1-q) b \sum_{n=0}^{\infty} f\left(b q^{n}\right) q^{n}
$$

provided the sum converge absolutely. 
The $q$-Jackson integral in a generic interval $[a, b]$ is given by [5]

$$
\int_{a}^{b} f(x) d_{q} x=\int_{0}^{b} f(x) d_{q} x-\int_{0}^{a} f(x) d_{q} x .
$$

In [10], the authors presented a Riemann-type $q$-integral by:

$$
R_{q}(f ; a, b)=(b-a)(1-q) \sum_{k=0}^{\infty} f\left(a+(b-a) q^{k}\right) q^{k} .
$$

We can get another definition from the Riemman-type $q$-integral:

$$
\begin{aligned}
& \frac{2}{b-a} \int_{a}^{b} f(x) d_{q}^{R} x \\
= & (1-q) \sum_{k=0}^{\infty}\left(f\left(\frac{a+b}{2}+q^{k}\left(\frac{b-a}{2}\right)\right)+f\left(\frac{a+b}{2}-q^{k}\left(\frac{b-a}{2}\right)\right)\right) q^{k}
\end{aligned}
$$

From the $q$-Jackson integral we can write:

$$
\begin{aligned}
\frac{2}{b-a} \int_{a}^{b} f(x) d_{q}^{R} x & =\int_{-1}^{1} f\left(\frac{1-t}{2} a+\frac{1+t}{2} b\right) d_{q} t \\
& =\int_{-1}^{1} f\left(\frac{1+t}{2} a+\frac{1-t}{2} b\right) d_{q} t .
\end{aligned}
$$

Contrary to the $q$-Jackson integral, if

$$
f(x) \leq g(x), \quad x \in[a, b]
$$

then

$$
\int_{a}^{b} f(x) d_{q}^{R} x \leq \int_{a}^{b} g(x) d_{q}^{R} x .
$$

In [11], the authors established the $q$-analogue of Hermite-Hadamard inequalities for convex function

Theorem 1.10. Let $f:[a, b] \rightarrow \mathbb{R}$ be a convex function. Then one has the inequalities:

$$
f\left(\frac{a+b}{2}\right) \leq \frac{1}{b-a} \int_{a}^{b} f(t) d_{q}^{R} t \leq \frac{f(a)+f(b)}{2} .
$$

For some recent studies on quantum integral inequalities, see [7, 8, 9].

\section{Main results}

In this section, we discuss main results of the paper. For this we need the following Lemma:

Lemma 2.1. If $f:[a, b] \rightarrow \mathbb{R}$ is a convex function. Then the following inequality holds for all $s, t, u, v \in[0,1]$ with $s \leq t \leq u \leq v$ and $t+u=s+v$

$$
f(t)+f(u) \leq f(s)+f(v)
$$


Proof. Since $f$ is convex function, for all $s, t, u, v \in[0,1]$ with $s \leq t \leq u \leq v$ and $t+u=s+v$, we obtain

$$
\frac{f(v)-f(u)}{v-u} \geq \frac{f(u)-f(t)}{u-t} \geq \frac{f(t)-f(s)}{t-s}=\frac{f(t)-f(s)}{v-u}
$$

then, we have

$$
f(v)-f(u) \geq f(t)-f(s)
$$

The proof is completed.

Theorem 2.2. Let $f:[a, b] \rightarrow \mathbb{R}$ be a convex function and $p:[a, b] \rightarrow \mathbb{R}$ be a nonnegative, integrable and symmetric about $x=\frac{a+b}{2}$. If $H$ and $F$ are defined on $[0,1]$ by

$$
H(t)=\int_{a}^{b} f\left(t x+(1-t) \frac{a+b}{2}\right) p(x) d_{q}^{R} x
$$

and

$F(t)=\int_{a}^{b} \frac{1}{2}\left[f\left(\frac{1+t}{2} a+\frac{1-t}{2} x\right) p\left(\frac{x+a}{2}\right)+f\left(\frac{1+t}{2} b+\frac{1-t}{2} x\right) p\left(\frac{x+b}{2}\right)\right] d_{q}^{R} x$,

then $H, F$ are convex and increasing on $[0,1]$ and for all $t \in[0,1]$

$$
f\left(\frac{a+b}{2}\right) \int_{a}^{b} p(x) d_{q}^{R} x=H(0) \leq H(t) \leq H(1)=\int_{a}^{b} f(x) p(x) d_{q}^{R} x
$$

and

$$
\int_{a}^{b} f(x) p(x) d_{q}^{R} x=F(0) \leq F(t) \leq F(1)=\frac{f(a)+f(b)}{2} \int_{a}^{b} p(x) d_{q}^{R} x .
$$

Proof. For all $t_{1}, t_{2}, \lambda \in[0,1]$ and $x \in[a, b]$, by convexity of $f$, we have

$$
\begin{aligned}
& f\left(\left(\lambda t_{1}+(1-\lambda) t_{2}\right) x+\left(1-\left(\lambda t_{1}+(1-\lambda) t_{2}\right)\right) \frac{a+b}{2}\right) \\
= & f\left(\lambda\left(t_{1} x+\left(1-t_{1}\right) \frac{a+b}{2}\right)+(1-\lambda)\left(t_{2} x+\left(1-t_{2}\right) \frac{a+b}{2}\right)\right) \\
\leq & \lambda f\left(t_{1} x+\left(1-t_{1}\right) \frac{a+b}{2}\right)+(1-\lambda) f\left(t_{2} x+\left(1-t_{2}\right) \frac{a+b}{2}\right) .
\end{aligned}
$$

Utilizing the inequality (1.13) and by $p(\cdot)$ a nonnegative function, we get

$$
\begin{aligned}
& H\left(\lambda t_{1}+(1-\lambda) t_{2}\right) \\
= & \int_{a}^{b} f\left(\left(\lambda t_{1}+(1-\lambda) t_{2}\right) x+\left(1-\left(\lambda t_{1}+(1-\lambda) t_{2}\right)\right) \frac{a+b}{2}\right) p(x) d_{q}^{R} x \\
\leq & \lambda \int_{a}^{b} f\left(t_{1} x+\left(1-t_{1}\right) \frac{a+b}{2}\right) p(x) d_{q}^{R} x \\
+ & (1-\lambda) \int_{a}^{b} f\left(t_{2} x+\left(1-t_{2}\right) \frac{a+b}{2}\right) p(x) d_{q}^{R} x \\
= & \lambda H\left(t_{1}\right)+(1-\lambda) H\left(t_{2}\right),
\end{aligned}
$$


which shows that $H$ is convex on $[0,1]$.

Now, let $0 \leq s \leq t \leq 1$ and $w \in[0,1]$, we have

$$
\begin{aligned}
& t\left(a+b-\left(\frac{1-w}{2} a+\frac{1+w}{2} b\right)\right)+(1-t) \frac{a+b}{2} \\
\leq & s\left(a+b-\left(\frac{1-w}{2} a+\frac{1+w}{2} b\right)\right)+(1-s) \frac{a+b}{2} \\
\leq & s\left(\frac{1-w}{2} a+\frac{1+w}{2} b\right)+(1-s) \frac{a+b}{2} \\
\leq & t\left(\frac{1-w}{2} a+\frac{1+w}{2} b\right)+(1-t) \frac{a+b}{2},
\end{aligned}
$$

and for all $w \in[-1,0]$, we have

$$
\begin{aligned}
& t\left(\frac{1-w}{2} a+\frac{1+w}{2} b\right)+(1-t) \frac{a+b}{2} \\
\leq & s\left(\frac{1-w}{2} a+\frac{1+w}{2} b\right)+(1-s) \frac{a+b}{2} \\
\leq & s\left(a+b-\left(\frac{1-w}{2} a+\frac{1+w}{2} b\right)\right)+(1-s) \frac{a+b}{2} \\
\leq & t\left(a+b-\left(\frac{1-w}{2} a+\frac{1+w}{2} b\right)\right)+(1-t) \frac{a+b}{2},
\end{aligned}
$$

for all $w \in[-1,1]$, we get

$$
\begin{aligned}
& \left(s\left(a+b-\left(\frac{1-w}{2} a+\frac{1+w}{2} b\right)\right)+(1-s) \frac{a+b}{2}\right) \\
+ & \left(s\left(\frac{1-w}{2} a+\frac{1+w}{2} b\right)+(1-s) \frac{a+b}{2}\right) \\
= & \left(t\left(\frac{1-w}{2} a+\frac{1+w}{2} b\right)+(1-t) \frac{a+b}{2}\right) \\
+ & \left(t\left(a+b-\left(\frac{1-w}{2} a+\frac{1+w}{2} b\right)\right)+(1-t) \frac{a+b}{2}\right) .
\end{aligned}
$$

By Lemma 2.1, we have

$$
\begin{aligned}
& f\left(s\left(a+b-\left(\frac{1-w}{2} a+\frac{1+w}{2} b\right)\right)+(1-s) \frac{a+b}{2}\right) \\
+ & f\left(s\left(\frac{1-w}{2} a+\frac{1+w}{2} b\right)+(1-s) \frac{a+b}{2}\right) \\
\leq & f\left(t\left(\frac{1-w}{2} a+\frac{1+w}{2} b\right)+(1-t) \frac{a+b}{2}\right) \\
+ & f\left(t\left(a+b-\left(\frac{1-w}{2} a+\frac{1+w}{2} b\right)\right)+(1-t) \frac{a+b}{2}\right) .
\end{aligned}
$$


Then,

$$
\begin{aligned}
H(s) & =\int_{a}^{b} f\left(s x+(1-s) \frac{a+b}{2}\right) p(x) d_{q}^{R} x \\
& =\frac{b-a}{2} \int_{-1}^{1} f\left(s\left(\frac{1-w}{2} a+\frac{1+w}{2} b\right)+(1-s) \frac{a+b}{2}\right) \\
& \times p\left(\frac{1-w}{2} a+\frac{1+w}{2} b\right) d_{q} w \\
& =\frac{b-a}{4} \int_{-1}^{1} f\left(s\left(\frac{1-w}{2} a+\frac{1+w}{2} b\right)+(1-s) \frac{a+b}{2}\right) \\
& \times p\left(\frac{1-w}{2} a+\frac{1+w}{2} b\right) d_{q} w \\
& +\frac{b-a}{4} \int_{-1}^{1} f\left(s\left(\frac{1-w}{2} a+\frac{1+w}{2} b\right)+(1-s) \frac{a+b}{2}\right) \\
& \times p\left(\frac{1-w}{2} a+\frac{1+w}{2} b\right) d_{q} w .
\end{aligned}
$$

Since $p(\cdot)$ is nonnegative, integrable and symmetric about $x=\frac{a+b}{2}$, we get

$$
\begin{aligned}
& \frac{b-a}{4} \int_{-1}^{1} f\left(s\left(\frac{1-w}{2} a+\frac{1+w}{2} b\right)+(1-s) \frac{a+b}{2}\right) \\
\times & p\left(\frac{1-w}{2} a+\frac{1+w}{2} b\right) d_{q} w \\
= & \frac{b-a}{4} \int_{-1}^{1} f\left(s\left(\frac{1-w}{2} a+\frac{1+w}{2} b\right)+(1-s) \frac{a+b}{2}\right) \\
\times & p\left(a+b-\left(\frac{1-w}{2} a+\frac{1+w}{2} b\right)\right) d_{q} w \\
= & \frac{b-a}{4} \int_{-1}^{1} f\left(s\left(\frac{1-w}{2} a+\frac{1+w}{2} b\right)+(1-s) \frac{a+b}{2}\right) \\
\times & p\left(\frac{1+w}{2} a+\frac{1-w}{2} b\right) d_{q} w \\
= & \frac{b-a}{4} \int_{-1}^{1} f\left(s\left(\frac{1+w}{2} a+\frac{1-w}{2} b\right)+(1-s) \frac{a+b}{2}\right) \\
\times & p\left(\frac{1-w}{2} a+\frac{1+w}{2} b\right) d_{q} w \\
= & \frac{b-a}{4} \int_{-1}^{1} f\left(s\left(a+b-\left(\frac{1-w}{2} a+\frac{1+w}{2} b\right)\right)+(1-s) \frac{a+b}{2}\right) \\
\times & p\left(\frac{1-w}{2} a+\frac{1+w}{2} b\right) d_{q} w,
\end{aligned}
$$


then, we obtain

$$
\begin{aligned}
H(s) & =\frac{b-a}{4} \int_{-1}^{1} f\left(s\left(a+b-\left(\frac{1-w}{2} a+\frac{1+w}{2} b\right)\right)+(1-s) \frac{a+b}{2}\right) \\
& \times p\left(\frac{1-w}{2} a+\frac{1+w}{2} b\right) d_{q} w \\
& +\frac{b-a}{4} \int_{-1}^{1} f\left(s\left(\frac{1-w}{2} a+\frac{1+w}{2} b\right)+(1-s) \frac{a+b}{2}\right) \\
& \times p\left(\frac{1-w}{2} a+\frac{1+w}{2} b\right) d_{q} w \\
& \leq \frac{b-a}{4} \int_{-1}^{1} f\left(t\left(\frac{1-w}{2} a+\frac{1+w}{2} b\right)+(1-t) \frac{a+b}{2}\right) \\
& \times p\left(\frac{1-w}{2} a+\frac{1+w}{2} b\right) d_{q} w \\
& +\frac{b-a}{4} \int_{-1}^{1} f\left(t\left(a+b-\left(\frac{1-w}{2} a+\frac{1+w}{2} b\right)\right)+(1-t) \frac{a+b}{2}\right) \\
& \times p\left(\frac{1-w}{2} a+\frac{1+w}{2} b\right) d_{q} w \\
& =H(t) .
\end{aligned}
$$

Thus, $H$ is increasing on $[0,1]$ and the inequality (2.2) holds for all $t \in[0,1]$.

For all $t_{1}, t_{2}, \lambda \in[0,1]$ and $x \in[a, b]$, by convexity of $f$, we get

$$
\begin{aligned}
& f\left(\frac{1+\lambda t_{1}+(1-\lambda) t_{2}}{2} a+\frac{1-\lambda t_{1}-(1-\lambda) t_{2}}{2} x\right) \\
= & f\left(\frac{\lambda\left(1+t_{1}\right)+(1-\lambda)\left(1+t_{2}\right)}{2} a+\frac{\lambda\left(1-t_{1}\right)+(1-\lambda)\left(1-t_{2}\right)}{2} x\right) \\
\leq & \lambda f\left(\frac{\left(1+t_{1}\right)}{2} a+\frac{\left(1-t_{1}\right)}{2} x\right)+(1-\lambda) f\left(\frac{\left(1+t_{2}\right)}{2} a+\frac{\left(1-t_{2}\right)}{2} x\right) .
\end{aligned}
$$

Similarly, we have

$$
\begin{aligned}
& f\left(\frac{1+\lambda t_{1}+(1-\lambda) t_{2}}{2} b+\frac{1-\lambda t_{1}-(1-\lambda) t_{2}}{2} x\right) \\
\leq & \lambda f\left(\frac{\left(1+t_{1}\right)}{2} b+\frac{\left(1-t_{1}\right)}{2} x\right)+(1-\lambda) f\left(\frac{\left(1+t_{2}\right)}{2} b+\frac{\left(1-t_{2}\right)}{2} x\right),
\end{aligned}
$$


then, using $(2.5),(2.6),(1.13)$ and the fact that $p(\cdot)$ is nonnegative function, we obtain

$$
\begin{aligned}
& F\left(\lambda t_{1}+(1-\lambda) t_{2}\right) \\
\leq & \lambda \int_{a}^{b} f\left(\frac{1+t_{1}}{2} a+\frac{1-t_{1}}{2} x\right) p\left(\frac{x+a}{2}\right) d_{q}^{R} x \\
+ & (1-\lambda) \int_{a}^{b} f\left(\frac{1+t_{2}}{2} a+\frac{1-t_{2}}{2} x\right) p\left(\frac{x+a}{2}\right) d_{q}^{R} x \\
+ & \lambda \int_{a}^{b} f\left(\frac{1+t_{1}}{2} b+\frac{1-t_{1}}{2} x\right) p\left(\frac{x+b}{2}\right) d_{q}^{R} x \\
+ & (1-\lambda) \int_{a}^{b} f\left(\frac{1+t_{2}}{2} b+\frac{1-t_{2}}{2} x\right) p\left(\frac{x+b}{2}\right) d_{q}^{R} x \\
= & \lambda F\left(t_{1}\right)+(1-\lambda) F\left(t_{2}\right) .
\end{aligned}
$$

Thus, $F$ is convex on $[0,1]$.

For all $w \in[-1,1]$, and $0 \leq s \leq t \leq 0$, we have

$$
\begin{aligned}
& \frac{1+t}{2} a+\frac{1-t}{2}\left(\frac{1-w}{2} a+\frac{1+w}{2} b\right) \\
\leq & \frac{1+s}{2} a+\frac{1-s}{2}\left(\frac{1-w}{2} a+\frac{1+w}{2} b\right) \\
\leq & \frac{1+s}{2} b+\frac{1-s}{2}\left(a+b-\left(\frac{1-w}{2} a+\frac{1+w}{2} b\right)\right) \\
\leq & \frac{1+t}{2} b+\frac{1-t}{2}\left(a+b-\left(\frac{1-w}{2} a+\frac{1+w}{2} b\right)\right),
\end{aligned}
$$

where

$$
\begin{aligned}
& \frac{1+s}{2} a+\frac{1-s}{2}\left(\frac{1-w}{2} a+\frac{1+w}{2} b\right) \\
+ & \frac{1+s}{2} b+\frac{1-s}{2}\left(a+b-\left(\frac{1-w}{2} a+\frac{1+w}{2} b\right)\right) \\
= & \frac{1+t}{2} a+\frac{1-t}{2}\left(\frac{1-w}{2} a+\frac{1+w}{2} b\right) \\
+ & \frac{1+t}{2} b+\frac{1-t}{2}\left(a+b-\left(\frac{1-w}{2} a+\frac{1+w}{2} b\right)\right) .
\end{aligned}
$$


Using Lemma 2.1, and the fact that $p(\cdot)$ is nonnegative function, we have

$$
\begin{aligned}
& f\left(\frac{1+s}{2} a+\frac{1-s}{2}\left(\frac{1-w}{2} a+\frac{1+w}{2} b\right)\right) \\
\times & p\left(\frac{1}{2}\left(a+\frac{1-w}{2} a+\frac{1+w}{2} b\right)\right) \\
+ & f\left(\frac{1+s}{2} b+\frac{1-s}{2}\left(a+b-\left(\frac{1-w}{2} a+\frac{1+w}{2} b\right)\right)\right) \\
\times & p\left(\frac{1}{2}\left(a+\frac{1-w}{2} a+\frac{1+w}{2} b\right)\right) \\
\leq & f\left(\frac{1+t}{2} a+\frac{1-t}{2}\left(\frac{1-w}{2} a+\frac{1+w}{2} b\right)\right) \\
\times & p\left(\frac{1}{2}\left(a+\frac{1-w}{2} a+\frac{1+w}{2} b\right)\right) \\
+ & f\left(\frac{1+t}{2} b+\frac{1-t}{2}\left(a+b-\left(\frac{1-w}{2} a+\frac{1+w}{2} b\right)\right)\right) \\
\times & p\left(\frac{1}{2}\left(a+\frac{1-w}{2} a+\frac{1+w}{2} b\right)\right) .
\end{aligned}
$$

Integrating with respect to $w$ on $[-1,1]$, we have

$$
\begin{aligned}
& \int_{-1}^{1} f\left(\frac{1+s}{2} a+\frac{1-s}{2}\left(\frac{1-w}{2} a+\frac{1+w}{2} b\right)\right) \\
\times & p\left(\frac{1}{2}\left(a+\frac{1-w}{2} a+\frac{1+w}{2} b\right)\right) d_{q} w \\
+ & \int_{-1}^{1} f\left(\frac{1+s}{2} b+\frac{1-s}{2}\left(a+b-\left(\frac{1-w}{2} a+\frac{1+w}{2} b\right)\right)\right) \\
\times & p\left(\frac{1}{2}\left(a+\frac{1-w}{2} a+\frac{1+w}{2} b\right)\right) d_{q} w \\
\leq & \int_{-1}^{1} f\left(\frac{1+t}{2} a+\frac{1-t}{2}\left(\frac{1-w}{2} a+\frac{1+w}{2} b\right)\right) \\
\times & p\left(\frac{1}{2}\left(a+\frac{1-w}{2} a+\frac{1+w}{2} b\right)\right) d_{q} w \\
+ & \int_{-1}^{1} f\left(\frac{1+t}{2} b+\frac{1-t}{2}\left(a+b-\left(\frac{1-w}{2} a+\frac{1+w}{2} b\right)\right)\right) \\
\times & p\left(\frac{1}{2}\left(a+\frac{1-w}{2} a+\frac{1+w}{2} b\right)\right) d_{q} w,
\end{aligned}
$$


where, using the fact that $p(\cdot)$ is nonnegative, integrable and symmetric about $x=\frac{a+b}{2}$, we get

$$
\begin{aligned}
& \int_{-1}^{1} f\left(\frac{1+s}{2} b+\frac{1-s}{2}\left(a+b-\left(\frac{1-w}{2} a+\frac{1+w}{2} b\right)\right)\right) \\
\times & p\left(\frac{1}{2}\left(a+\frac{1-w}{2} a+\frac{1+w}{2} b\right)\right) d_{q} w \\
= & \int_{-1}^{1} f\left(\frac{1+s}{2} b+\frac{1-s}{2}\left(a+b-\left(\frac{1-w}{2} a+\frac{1+w}{2} b\right)\right)\right) \\
\times & p\left(a+b-\frac{1}{2}\left(a+\frac{1-w}{2} a+\frac{1+w}{2} b\right)\right) d_{q} w \\
= & \int_{-1}^{1} f\left(\frac{1+s}{2} b+\frac{1-s}{2}\left(\frac{1+w}{2} a+\frac{1-w}{2} b\right)\right) \\
\times & p\left(\frac{1}{2}\left(b+\frac{1+w}{2} a+\frac{1-w}{2} b\right)\right) d_{q} w \\
= & \int_{a}^{b} f\left(\frac{1+s}{2} b+\frac{1-s}{2} x\right) p\left(\frac{b+x}{2}\right) d_{q}^{R} x .
\end{aligned}
$$

Similarly, we have

$$
\begin{aligned}
& \int_{-1}^{1} f\left(\frac{1+t}{2} b+\frac{1-t}{2}\left(a+b-\left(\frac{1-w}{2} a+\frac{1+w}{2} b\right)\right)\right) \\
\times & p\left(\frac{1}{2}\left(a+\frac{1-w}{2} a+\frac{1+w}{2} b\right)\right) d_{q} w \\
= & \int_{a}^{b} f\left(\frac{1+t}{2} a+\frac{1-t}{2} x\right) p\left(\frac{b+x}{2}\right) d_{q}^{R} x,
\end{aligned}
$$

then,

$$
\begin{aligned}
F(s) & =\frac{1}{2} \int_{a}^{b} \frac{1}{2} f\left(\frac{1+s}{2} a+\frac{1-s}{2} x\right) \\
& \times p\left(\frac{a+x}{2}\right) d_{q}^{R} x+\int_{a}^{b} \frac{1}{2} f\left(\frac{1+s}{2} b+\frac{1-s}{2} x\right) p\left(\frac{b+x}{2}\right) d_{q}^{R} x \\
& \leq \int_{a}^{b} \frac{1}{2} f\left(\frac{1+t}{2} a+\frac{1-t}{2} x\right) \\
& \times p\left(\frac{a+x}{2}\right) d_{q}^{R} x+\int_{a}^{b} \frac{1}{2} f\left(\frac{1+t}{2} b+\frac{1-t}{2} x\right) p\left(\frac{b+x}{2}\right) d_{q}^{R} x \\
& =F(t) .
\end{aligned}
$$

Thus, $F$ is increasing on $[0,1]$ and the inequality (2.3) holds for all $t \in[0,1]$. 
Theorem 2.3. Let $f:[a, b] \rightarrow \mathbb{R}$ be a h-convex function and $p:[a, b] \rightarrow \mathbb{R}$ be positive, integrable, and symmetric about $x=\frac{a+b}{2}$. Then the following inequalities hold:

$$
\begin{aligned}
& \frac{1}{2 h\left(\frac{1}{2}\right)} f\left(\frac{a+b}{2}\right) \int_{a}^{b} p(x) d_{q}^{R} x \\
\leq & \int_{a}^{b} f(x) p(x) d_{q}^{R} x \\
\leq & f(a) \int_{a}^{b} h\left(\frac{b-x}{b-a}\right) p(x) d_{q}^{R} x+f(b) \int_{a}^{b} h\left(\frac{x-a}{b-a}\right) p(x) d_{q}^{R} x
\end{aligned}
$$

Proof. Since $f$ is $h$-convex function, we have

$$
f\left(\frac{x+y}{2}\right) \leq h\left(\frac{1}{2}\right)(f(x)+f(y))
$$

for all $x, y \in[a, b]$.

In $(2.7)$, if we choose $x=\frac{1-w}{2} a+\frac{1+w}{2} b$ and $y=\frac{1+w}{2} a+\frac{1-w}{2} b, w \in[-1,1]$ and by $p$ is positive, we get

$$
\begin{aligned}
& f\left(\frac{a+b}{2}\right) p\left(\frac{1-w}{2} a+\frac{1+w}{2} b\right) \\
= & f\left(\frac{1}{2}\left(\frac{1-w}{2} a+\frac{1+w}{2} b\right)+\frac{1}{2}\left(\frac{1+w}{2} a+\frac{1-w}{2} b\right)\right) p\left(\frac{1-w}{2} a+\frac{1+w}{2} b\right) \\
\leq & h\left(\frac{1}{2}\right) f\left(\frac{1-w}{2} a+\frac{1+w}{2} b\right) p\left(\frac{1-w}{2} a+\frac{1+w}{2} b\right) \\
+ & h\left(\frac{1}{2}\right) f\left(\frac{1+w}{2} a+\frac{1-w}{2} b\right) p\left(\frac{1-w}{2} a+\frac{1+w}{2} b\right)
\end{aligned}
$$

Integrating with respect $w$ over $[-1,1]$, we obtain

$$
\begin{aligned}
& f\left(\frac{a+b}{2}\right) \int_{-1}^{1} p\left(\frac{1-w}{2} a+\frac{1+w}{2} b\right) d_{q} w \\
\leq & h\left(\frac{1}{2}\right) \int_{-1}^{1} f\left(\frac{1-w}{2} a+\frac{1+w}{2} b\right) p\left(\frac{1-w}{2} a+\frac{1+w}{2} b\right) d_{q} w \\
+ & h\left(\frac{1}{2}\right) \int_{-1}^{1} f\left(\frac{1+w}{2} a+\frac{1-w}{2} b\right) p\left(\frac{1-w}{2} a+\frac{1+w}{2} b\right) d_{q} w
\end{aligned}
$$

Then, by $p$ is symmetric function about $\frac{a+b}{2}$, we have

$$
\begin{aligned}
& f\left(\frac{a+b}{2}\right) \frac{2}{b-a} \int_{a}^{b} p(x) d_{q}^{R} x \\
\leq & h\left(\frac{1}{2}\right) \frac{2}{b-a} \int_{a}^{b} f(x) p(x) d_{q}^{R} x+h\left(\frac{1}{2}\right) \frac{2}{b-a} \int_{a}^{b} f(x) p\left(\frac{a+b}{2}+\frac{a+b}{2}-x\right) d_{q}^{R} x \\
= & h\left(\frac{1}{2}\right) \frac{2}{b-a} \int_{a}^{b} f(x) p(x) d_{q}^{R} x+h\left(\frac{1}{2}\right) \frac{2}{b-a} \int_{a}^{b} f(x) p(x) d_{q}^{R} x,
\end{aligned}
$$

the first inequality is proved. 
The proof of second inequality is given as

$$
\begin{aligned}
& f\left(\frac{1-w}{2} a+\frac{1+w}{2} b\right) p\left(\frac{1-w}{2} a+\frac{1+w}{2} b\right) \\
\leq & f(a) h\left(\frac{1-w}{2}\right) p\left(\frac{1-w}{2} a+\frac{1+w}{2} b\right) \\
+ & f(b) h\left(\frac{1+w}{2}\right) p\left(\frac{1-w}{2} a+\frac{1+w}{2} b\right)
\end{aligned}
$$

we integrate $w$ on $[-1,1]$, we obtain

$$
\begin{aligned}
& \int_{-1}^{1} f\left(\frac{1-w}{2} a+\frac{1+w}{2} b\right) p\left(\frac{1-w}{2} a+\frac{1+w}{2} b\right) d_{q} w \\
\leq & \int_{-1}^{1} f(a) h\left(\frac{1-w}{2}\right) p\left(\frac{1-w}{2} a+\frac{1+w}{2} b\right) d_{q} w \\
+ & \int_{-1}^{1} f(b) h\left(\frac{1+w}{2}\right) p\left(\frac{1-w}{2} a+\frac{1+w}{2} b\right) d_{q} w .
\end{aligned}
$$

Then

$$
\begin{aligned}
\frac{2}{b-a} \int_{a}^{b} f(x) p(x) d_{q}^{R} x & \leq f(a) \frac{2}{b-a} \int_{a}^{b} h\left(\frac{b-x}{b-a}\right) p(x) d_{q}^{R} x \\
& +\frac{2}{b-a} f(b) \int_{a}^{b} h\left(\frac{x-a}{b-a}\right) p(x) d_{q}^{R} x .
\end{aligned}
$$

The proof is complete.

Remark 2.4. If we choose $p(x)=1$ and $h(t)=t$, then Theorem(2.3) reduces to Theorem(1.10).

Corollary 2.5. Let $f \in K_{s}^{2}, a, b \in[0, \infty[$ with $a<b$ and $p:[a, b] \rightarrow \mathbb{R}$ is nonnegative, integrable and symmetric about $\frac{a+b}{2}$. Then

$$
\begin{aligned}
& 2^{s-1} f\left(\frac{a+b}{2}\right) \int_{a}^{b} p(x) d_{q}^{R} x \leq \int_{a}^{b} f(x) p(x) d_{q}^{R} x \\
\leq & f(a) \int_{a}^{b}\left(\frac{b-x}{b-a}\right)^{s} p(x) d_{q}^{R} x+f(b) \int_{a}^{b}\left(\frac{x-a}{b-a}\right)^{s} p(x) d_{q}^{R} x
\end{aligned}
$$

Remark 2.6. If we choose $s=1, h(t)=t$ and $p(x)=1$, then Corollary (2.5) reduces to Theorem(1.10).

Theorem 2.7. Let $f:[0, \infty[\rightarrow \mathbb{R}$ be an m-convex function with $m \in] 0,1]$. If $0 \leq a<$ $b<\infty$ and $f \in L_{1}[0, b]$, then

$$
\int_{a}^{b} f(x) p(x) d_{q}^{R} x \leq \min \left\{\int_{a}^{b} L_{x}(a, b) p(x) d_{q}^{R} x, \int_{a}^{b} L_{x}(b, a) p(x) d_{q}^{R} x\right\} .
$$

with $L_{x}(a, b)=\left(f(a)\left(\frac{b-x}{b-a}\right)+m f\left(\frac{b}{m}\right)\left(\frac{x-a}{b-a}\right)\right)$. 
Proof. Since $f$ is $m$-convex and for all $w \in[-1,1]$, we have

$$
\begin{aligned}
& f\left(\frac{1-w}{2} a+m \frac{1+w}{2 m} b\right) p\left(\frac{1-w}{2} a+\frac{1+w}{2} b\right) \\
\leq & \left(\frac{1-w}{2}\right) f(a) p\left(\frac{1-w}{2} a+\frac{1+w}{2} b\right) \\
+ & m\left(\frac{1+w}{2}\right) f\left(\frac{b}{m}\right) p\left(\frac{1-w}{2} a+\frac{1+w}{2} b\right),
\end{aligned}
$$

and

$$
\begin{aligned}
& f\left(\frac{1-w}{2} b+m \frac{1+w}{2 m} a\right) p\left(\frac{1-w}{2} b+\frac{1+w}{2} a\right) \\
\leq & \left(\frac{1-w}{2}\right) f(b) p\left(\frac{1-w}{2} b+\frac{1+w}{2} a\right) \\
+ & m\left(\frac{1+w}{2}\right) f\left(\frac{a}{m}\right) p\left(\frac{1-w}{2} b+\frac{1+w}{2} a\right) .
\end{aligned}
$$

Then, by integrating both sides with respect $w$ on $[-1,1]$, we have

$$
\begin{aligned}
\frac{2}{b-a} \int_{a}^{b} f(x) p(x) d_{q}^{R} x & \leq f(a) \frac{2}{b-a} \int_{a}^{b}\left(\frac{b-x}{b-a}\right) p(x) d_{q}^{R} x \\
& +m f\left(\frac{b}{m}\right) \frac{2}{b-a} \int_{a}^{b}\left(\frac{x-a}{b-a}\right) p(x) d_{q}^{R} x
\end{aligned}
$$

and

$$
\begin{aligned}
\frac{2}{b-a} \int_{a}^{b} f(x) p(x) d_{q}^{R} x & \leq f(b) \frac{2}{b-a} \int_{a}^{b}\left(\frac{a-x}{a-b}\right) p(x) d_{q}^{R} x \\
& +m f\left(\frac{a}{m}\right) \frac{2}{b-a} \int_{a}^{b}\left(\frac{x-b}{a-b}\right) p(x) d_{q}^{R} x
\end{aligned}
$$

This completes the proof.

Theorem 2.8. Let $f:[0, \infty[\rightarrow \mathbb{R}$ be a m-convex function with $m \in] 0,1]$ and $p:[a, b] \rightarrow$ $\mathbb{R}$ is nonnegative, integrable and symmetric about $\frac{a+b}{2}$. Then

$$
\begin{aligned}
2 f\left(\frac{a+b}{2}\right) \int_{a}^{b} p(x) d_{q}^{R} x & \leq \int_{a}^{b}\left(f(x)+m f\left(\frac{x}{m}\right)\right) p(x) d_{q}^{R} x \\
& \leq\left(f(a)+m f\left(\frac{a}{m}\right)\right) \int_{a}^{b}\left(\frac{b-x}{b-a}\right) p(x) d_{q}^{R} x \\
& +\left(m f\left(\frac{b}{m}\right)+m^{2} f\left(\frac{b}{m^{2}}\right)\right) \int_{a}^{b}\left(\frac{x-a}{b-a}\right) p(x) d_{q}^{R} x .
\end{aligned}
$$


Proof. According to the definition of $m$-convex function, for all $t \in[-1,1]$, we have

$$
\begin{aligned}
& f\left(\frac{a+b}{2}\right) p\left(\frac{1-t}{2} a+\frac{1+t}{2} b\right) \\
= & f\left(\frac{1}{2}\left(\frac{1-t}{2} a+\frac{1+t}{2} b\right)+\frac{m}{2 m}\left(\frac{1+t}{2} a+\frac{1-t}{2} b\right)\right) p\left(\frac{1-t}{2} a+\frac{1+t}{2} b\right) \\
\leq & \frac{1}{2} f\left(\frac{1-t}{2} a+\frac{1+t}{2} b\right) p\left(\frac{1-t}{2} a+\frac{1+t}{2} b\right) \\
+ & \frac{m}{2} f\left(\frac{\frac{1+t}{2} a+\frac{1-t}{2} b}{m}\right) p\left(\frac{1-t}{2} a+\frac{1+t}{2} b\right) .
\end{aligned}
$$

Integrating with respect to $t$ on $[-1,1]$, we have

$$
\begin{aligned}
& \int_{-1}^{1} f\left(\frac{a+b}{2}\right) p\left(\frac{1-t}{2} a+\frac{1+t}{2} b\right) d_{q} t \\
\leq & \frac{1}{2} \int_{-1}^{1} f\left(\frac{1-t}{2} a+\frac{1+t}{2} b\right) p\left(\frac{1-t}{2} a+\frac{1+t}{2} b\right) d_{q} t \\
+ & \int_{-1}^{1} \frac{m}{2} f\left(\frac{\frac{1+t}{2} a+\frac{1-t}{2} b}{m}\right) p\left(\frac{1-t}{2} a+\frac{1+t}{2} b\right) d_{q} t .
\end{aligned}
$$

Since $p(\cdot)$ is symmetric function, so, we have

$$
\begin{aligned}
& f\left(\frac{a+b}{2}\right) \frac{2}{b-a} \int_{a}^{b} p(x) d_{q}^{R} x \\
\leq & \frac{2}{2(b-a)} \int_{a}^{b} f(x) p(x) d_{q}^{R} x+\frac{2 m}{2(b-a)} \int_{a}^{b} f\left(\frac{x}{m}\right) p(a+b-x) d_{q}^{R} x \\
= & \frac{1}{(b-a)} \int_{a}^{b} f(x) p(x) d_{q}^{R} x+\frac{m}{(b-a)} \int_{a}^{b} f\left(\frac{x}{m}\right) p(x) d_{q}^{R} x .
\end{aligned}
$$

Then

$$
f\left(\frac{a+b}{2}\right) 2 \int_{a}^{b} p(x) d_{q}^{R} x \leq \int_{a}^{b} f(x) p(x) d_{q}^{R} x+m \int_{a}^{b} f\left(\frac{x}{m}\right) p(x) d_{q}^{R} x .
$$

the first inequality is proved.

Now the proof of second inequality is given by

$$
\begin{aligned}
& f\left(\frac{1-t}{2} a+m \frac{1+t}{2 m} b\right) p\left(\frac{1-t}{2} a+\frac{1+t}{2} b\right) \\
\leq & f(a) \frac{1-t}{2} p\left(\frac{1-t}{2} a+\frac{1+t}{2} b\right) \\
+ & m \frac{1+t}{2} f\left(\frac{b}{m}\right) p\left(\frac{1-t}{2} a+\frac{1+t}{2} b\right),
\end{aligned}
$$


and

$$
\begin{aligned}
& m f\left(\frac{1-t}{2 m} a+\frac{1+t}{2 m^{2}} m b\right) p\left(\frac{1-t}{2} a+\frac{1+t}{2} b\right) \\
\leq & m f\left(\frac{a}{m}\right) \frac{1-t}{2} p\left(\frac{1-t}{2} a+\frac{1+t}{2} b\right) \\
+ & m^{2} \frac{1+t}{2} f\left(\frac{b}{m^{2}}\right) p\left(\frac{1-t}{2} a+\frac{1+t}{2} b\right) .
\end{aligned}
$$

Integrating both sides with respect to $t$ on $[-1,1]$, we obtain

$$
\begin{aligned}
\frac{2}{b-a} \int_{a}^{b} f(x) p(x) d_{q}^{R} x & \leq f(a) \frac{2}{b-a} \int_{a}^{b}\left(\frac{b-x}{b-a}\right) p(x) d_{q}^{R} x \\
& +f\left(\frac{b}{m}\right) \frac{2 m}{b-a} \int_{a}^{b}\left(\frac{x-a}{b-a}\right) p(x) d_{q}^{R} x,
\end{aligned}
$$

and

$$
\begin{aligned}
m \frac{2}{b-a} \int_{a}^{b} f\left(\frac{x}{m}\right) p(x) d_{q}^{R} x & \leq m f\left(\frac{a}{m}\right) \frac{2}{b-a} \int_{a}^{b} \frac{b-x}{b-a} p(x) d_{q}^{R} x \\
& +m^{2} f\left(\frac{b}{m^{2}}\right) \frac{2}{b-a} \int_{a}^{b} \frac{x-a}{b-a} p(x) d_{q}^{R} x
\end{aligned}
$$

Then, the proof of Theorem (2.8) is completed.

Theorem 2.9. Let $f:[0, \infty[\rightarrow \mathbb{R} \text { be an }(s, m) \text {-convex function with }(s, m) \in] 0,1]^{2}$ and $p:[a, b] \rightarrow \mathbb{R}$ is nonnegative, integrable and symmetric about $\frac{a+b}{2}$. Then

$$
\begin{aligned}
2^{s} f\left(\frac{a+b}{2}\right) \int_{a}^{b} p(x) d_{q}^{R} x & \leq \int_{a}^{b} f(x) p(x) d_{q}^{R} x+m\left(2^{s}-1\right) \int_{a}^{b} f\left(\frac{x}{m}\right) p(x) d_{q}^{R} x \\
& \leq\left(f(a)+m\left(2^{s}-1\right) f\left(\frac{a}{m}\right)\right) \int_{a}^{b}\left(\frac{b-x}{b-a}\right)^{s} p(x) d_{q}^{R} x \\
& +m\left(f\left(\frac{b}{m}\right)+m\left(2^{s}-1\right) f\left(\frac{b}{m^{2}}\right)\right) \int_{a}^{b}\left(\frac{x-a}{b-a}\right)^{s} p(x) d_{q}^{R} x
\end{aligned}
$$

Proof. Since $f$ is $(s, m)$-convex function on $[a, b]$, we can write

$$
\begin{aligned}
& f\left(\frac{1}{2}\left(\frac{1-t}{2} a+\frac{1+t}{2} b\right)+\frac{m}{2 m}\left(\frac{1+t}{2} a+\frac{1-t}{2} b\right)\right) p\left(\frac{1-t}{2} a+\frac{1+t}{2} b\right) \\
\leq & \left(\frac{1}{2}\right)^{s} f\left(\frac{1-t}{2} a+\frac{1+t}{2} b\right) p\left(\frac{1-t}{2} a+\frac{1+t}{2} b\right) \\
+ & m\left(1-\left(\frac{1}{2}\right)^{s}\right) f\left(\frac{1}{m}\left(\frac{1+t}{2} a+\frac{1-t}{2} b\right)\right) p\left(\frac{1-t}{2} a+\frac{1+t}{2} b\right) .
\end{aligned}
$$


Integrating with respect to $t$ on $[-1,1]$, we get

$$
\begin{aligned}
f\left(\frac{a+b}{2}\right) \frac{2}{b-a} \int_{a}^{b} p(x) d_{q}^{R} x & \leq\left(\frac{1}{2}\right)^{s} \frac{2}{b-a} \int_{a}^{b} f(x) p(x) d_{q}^{R} x \\
& \leq m\left(1-\left(\frac{1}{2}\right)^{s}\right) \frac{2}{b-a} \int_{a}^{b} f\left(\frac{x}{m}\right) p(a+b-x) d_{q}^{R} x .
\end{aligned}
$$

Since $p(\cdot)$ is symmetric function, so, we have

$$
2^{s} f\left(\frac{a+b}{2}\right) \int_{a}^{b} p(x) d_{q}^{R} x \leq \int_{a}^{b} f(x) p(x) d_{q}^{R} x+m\left(2^{s}-1\right) \int_{a}^{b} f\left(\frac{x}{m}\right) p(x) d_{q}^{R} x .
$$

Also, we have

$$
\begin{aligned}
& f\left(\frac{1-t}{2} a+\frac{1+t}{2} b\right) p\left(\frac{1-t}{2} a+\frac{1+t}{2} b\right) \\
= & f\left(\frac{1-t}{2} a+\frac{m}{m}\left(1-\left(\frac{1-t}{2}\right)\right) b\right) p\left(\frac{1-t}{2} a+\frac{1+t}{2} b\right) \\
\leq & \left(\frac{1-t}{2}\right)^{s} f(a) p\left(\frac{1-t}{2} a+\frac{1+t}{2} b\right) \\
+ & m\left(1-\left(\frac{1-t}{2}\right)^{s}\right) f\left(\frac{b}{m}\right) p\left(\frac{1-t}{2} a+\frac{1+t}{2} b\right),
\end{aligned}
$$

and

$$
\begin{aligned}
& f\left(\frac{\left(\frac{1-t}{2} a+\frac{1+t}{2} b\right)}{m}\right) p\left(\frac{1-t}{2} a+\frac{1+t}{2} b\right) \\
= & f\left(\frac{1-t}{2} \frac{a}{m}+\frac{m}{m^{2}}\left(1-\left(\frac{1-t}{2}\right)\right) b\right) p\left(\frac{1-t}{2} a+\frac{1+t}{2} b\right) \\
\leq & \left(\frac{1-t}{2}\right)^{s} f\left(\frac{a}{m}\right) p\left(\frac{1-t}{2} a+\frac{1+t}{2} b\right) \\
+ & m\left(1-\left(\frac{1-t}{2}\right)^{s}\right) f\left(\frac{b}{m^{2}}\right) p\left(\frac{1-t}{2} a+\frac{1+t}{2} b\right) .
\end{aligned}
$$

Integrating both sides with respect to $t$ on $[-1,1]$, we get

$$
\begin{aligned}
& \frac{2}{b-a} \int_{a}^{b} f(x) p(x) d_{q}^{R} x \\
\leq & \frac{2}{b-a} f(a) \int_{a}^{b}\left(\frac{b-x}{b-a}\right)^{s} p(x)+m f\left(\frac{b}{m}\right) \frac{2}{b-a} \int_{a}^{b}\left(1-\left(\frac{b-x}{b-a}\right)^{s}\right) p(x) d_{q}^{R} x,
\end{aligned}
$$

and

$$
\begin{aligned}
& \frac{2}{b-a} \int_{a}^{b} f\left(\frac{x}{m}\right) p(x) d_{q}^{R} x \\
\leq & \frac{2}{b-a} f\left(\frac{a}{m}\right) \int_{a}^{b}\left(\frac{b-x}{b-a}\right)^{s} p(x) d_{q}^{R} x+m f\left(\frac{b}{m^{2}}\right) \frac{2}{b-a} \int_{a}^{b}\left(1-\left(\frac{b-x}{b-a}\right)^{s}\right) p(x) d_{q}^{R} x .
\end{aligned}
$$

The proof of Theorem (2.9) is completed. 


\section{References}

[1] Breckner, W.W., Stetigkeitsaussagen fir eine Klasse verallgemeinerter konvexer funktionen in topologischen linearen Raumen, Publ. Inst. Math., 23(1978), 13-20.

[2] Féjer, L., Über die Fourierreihen II, (in Hungarian), Math. Naturwiss. Anz Ungar. Akad. Wiss., 24(1906), 369-390.

[3] Dragomir, S.S., Two mappings in connection to Hadamard's inequalities, J. Math. Anal. Appl., 167(1992), 49-56.

[4] Hudzik, H., Maligranda, L., Some remarks on s-convex functions, Aequationes Math., 48(1994), 100-111.

[5] Jackson, F.H., On a q-definite integrals, Quarterly J. Pure Appl. Math., 41(1910) 193203.

[6] Miheşan, V.G., A generalizations of the convexity, Seminar on Functional Equations, Approximation and Convexity, Cluj-Napoca, Romania, 1993.

[7] Noor, M.A., Noor, K.I., Awan, M.U., Some Quantum estimates for Hermite-Hadamard inequalities, Appl. Math. Comput., 251(2015), 675-679.

[8] Noor, M.A., Noor, K.I., Awan, M.U., Some quantum integral inequalities via preinvex functions, Appl. Math. Comput., 269(2015), 242-251.

[9] Noor, M.A., Noor, K.I., Awan, M.U., Quantum analogues of Hermite-Hadamard type inequalities for generalized convexity, in: N. Daras and M.T. Rassias (Ed.), Computation, Cryptography and Network Security, 2015.

[10] Rajković, P.M., Stanković, M.S., Marinković, S.D., The zeros of polynomials orthogonal with respect to q-integral on several intervals in the complex plane, Proceedings of The Fifth International Conference on Geometry, Integrability and Quantization, 2003, Varna, Bulgaria (ed. I.M. Mladenov, A.C. Hirsshfeld), 178-188.

[11] Taf, S., Brahim, K., Riahi, L., Some results for Hadamard-type inequalities in quantum calculs, Le Matematiche, 69(2014), no. 2, 243-258.

[12] Sarikaya, M.Z., On some new inequalities of Hadamard type involving h-convex functions, Acta Math. Univ. Comenianae, 79(2010), no. 2, 265-272.

[13] Toader, G.H., Some generalisations of the convexity, Proc. Colloq. Approx. Optim, (1984), 329-338.

[14] Tseng, K.L., Hwang, S.R., Dragomir, S.S., On some new inequalities of HermiteHadamard-Fejér type involving convex functions, Demonstratio Math., 40(2007), no. 1, 51-64.

[15] Varošanec, S., On h-convexity, J. Math. Anal. Appl., 326(2007), no. 1, 303-311.

[16] Yang, G.S., Tseng, K.L., A note On Hadamard's inequality, Tamkang. J. Math., 28(1997) 33-37.

[17] Yang, G.S., Tseng, K.L., On certain integral inequalities related to Hermite-Hadamard inequalites, J. Math. Anal. Appll, 239(1999), 180-187.

Kamel Brahim

Nabeul Preparatory Engineering Institute

Mrezgua, 8000 Nabeul, Tunisia

e-mail: Kamel.Brahim@ipeit.rnu.tn 


\section{Latifa Riahi}

Faculty of Science Mathematic, Physic and Naturelle of Tunis

e-mail: riahilatifa2013@gmail.com

\section{Muhammad Uzair Awan}

Department of Mathematic

Government College University, Faisalabad

e-mail: awan.uzair@gmail.com 